\title{
Análisis transcultural de factores motivacionales que influyen en la identidad del profesor
}

\begin{abstract}
Maria Cardelle-Elawar ${ }^{1}$, Leslie Irwin ${ }^{1}$, María Luisa Sanz de Acedo Lizarraga ${ }^{2}$
\end{abstract}

${ }^{1}$ College of Teacher Education and Leadership, Arizona State University, Glendale, (EEUU)

${ }^{2}$ Departamento de Psicología y Pedagogía, Universidad Pública de Navarra, Pamplona (España)

EEUU / España

Maria Cardelle-Elawar. 4701 West Thunderbird Road, College of Teacher Education and Leadership-Graduate Studies. Glendale, AZ 85306, USA. E-mail: mcardelle@asu.edu

(C) Education \& Psychology I+D+i and Editorial EOS (Spain) 


\section{Resumen}

Este estudio cualitativo describe los resultados de profesores de tres países distintos (Ghana, España, y EEUU) matriculados en una asignatura de psicología educativa de nivel posgrado, impartida por los autores. Un enfoque didáctica desde la teoría hacia la práctica fue empleada para motivar a los profesores que ejercen la profesión actualmente a que siguieran un proceso de investigación guiado por los profesores. Los participantes se implicaron en un proceso metacognitivo, autorregulado y narrativo-inquisitivo que les permitió situarse en un contextos educativos, históricos y políticos. Los participantes siguieron un protocolo de entrevista por parejas donde se preguntaron una serie de preguntas relacionadas con los motivos por los cuales se hicieron profesores en el pasado y qué les motiva a seguir ejerciendo en el presente. Textos narrativos significativos resumieron los resultados de las entrevistas y fueron analizados por temas generativos. Se discuten implicaciones para la enseñanza y la investigación, con referencias a prácticas de auto-regulación metacognitiva.

Palabras clave: análisis transcultural, motivación, identidad del profesor

Recepción: 25-07-07 Aceptación provisional: 31-08-07 Aceptación definitiva: 23-09-07 